\title{
Cross Surface Ambipolar Charge Percolation in Molecular Triads on Mesoscopic Oxide Films
}

Qing Wang, Shaik M. Zakeeruddin, Jens Cremer, ${ }^{\S}$ Peter Bäuerle, ${ }^{\S}$ Robin Humphry-Baker, and Michael Grätzel*

Laboratory for Photonics and Interfaces, Ecole Polytechnique Fédérale, CH-1015 Lausanne, Switzerland § Department of Organic Chemistry II, University of Ulm, 89081 Ulm, Germany

\section{Synthesis and characterization of PMI-T2-TPA}

\section{Di-tert-butyl 4,4'-[(4-\{5'-([N-(2,6-diisopropylphenyl)]-9-perylenyl-3,4-dicarboxyimide)-}

\section{3,4'-dihexyl-2,2'-bithien-5-yl\}phenyl)imino]dibenzoate (3):}

A solution of 1 (94 mg, $0.1 \mathrm{mmol})$ and the boronic ester 2 (69 mg, $0.12 \mathrm{mmol})$ in DME was degassed prior to the addition of a degassed $2 \mathrm{M}$ aqueous solution of tripotassium phosphate $(0.24 \mathrm{ml}, 0.48 \mathrm{mmol})$. Next, the catalyst $\left(\mathrm{Pd}_{2}(\mathrm{dba})_{3}[1.5 \mathrm{mg}, 2.5 \mu \mathrm{mol}],\left[\mathrm{HP}(t-\mathrm{Bu})_{3}\right] \mathrm{BF}_{4}[1.5\right.$ $\mathrm{mg}, 5.0 \mu \mathrm{mol}])$ was added. The resulting emulsion was carefully degassed and stirred at room temperature for 24 hours. Next, it was poured into water and the organic phase was separated while the aqueous phase was extracted with dichloromethane. The combined extracts were dried over magnesium sulphate and the solvent was removed by rotary evaporation. The crude product was purified by flash column chromatography $\left(\mathrm{SiO}_{2} /\right.$ petrol ether : ethyl acetate [5:1]) and was dried in vacuum, yielding $3(110 \mathrm{mg}, 87 \%)$ as a black solid. M.p.: $181-182^{\circ} \mathrm{C}$. 
${ }^{1} \mathrm{H}-\mathrm{NMR}\left(\mathrm{CDCl}_{3}\right): \delta=8.66(\mathrm{~d}, J=8.1 \mathrm{~Hz}, 2 \mathrm{H}$, Pery-1H,6H $), 8.49-8.42(\mathrm{~m}, 4 \mathrm{H}$, Pery- $2 H, 5 H, 7 H, 12 H$ ), 8.00 (d, $J=8.3 \mathrm{~Hz}, 1 \mathrm{H}$, Pery- $8 H$ ), 7.90 (approx. d, $J=8.8 \mathrm{~Hz}, 4 \mathrm{H}$, ( $t$-Bu)OOC-Ph-2H,6H), 7.69 (d, J=7.8 Hz, 1H,Pery-10H), 7.64 (t, J=8.0 Hz, 1H, Pery-11H), 7.55 (approx. d, $J=8.6 \mathrm{~Hz}, 2 \mathrm{H}, T P A-P h-3 H, 5 H), 7.48(\mathrm{t}, J=7.8 \mathrm{~Hz}, 1 \mathrm{H}, P h-4 H), 7.35$ (d, $J=7.8$ $\mathrm{Hz}, 2 \mathrm{H}, P h-3 H, 5 H), 7.18(\mathrm{~s}, 1 \mathrm{H}, T h-3 H), 7.17$ (s, 1H, Th'-4H), 7.15-7.09 (m, 6H, ( $t$-Bu)OOC-Ph-3H,5H, TPA-Ph-2H,6H), 2.86 (t, $J=7.8 \mathrm{~Hz}, 2 \mathrm{H}, T h-u^{\prime}-\mathrm{CH}_{2}$ ), 2.79 (hep, $J=6.8$ $\left.\mathrm{Hz}, 2 \mathrm{H}, P h-\mathrm{CH}-\left(\mathrm{CH}_{3}\right)_{2}\right), 2.45$ (t, J=7.5 Hz, 2H, Th- $\alpha-\mathrm{CH}_{2}$ ), 1.74 (quin, $J=6.8 \mathrm{~Hz}, 2 \mathrm{H}$, $T h-\beta$ ' $\left.-\mathrm{CH}_{2}\right), 1.59\left(\mathrm{~s}, 18 \mathrm{H}, \mathrm{C}-\left(\mathrm{CH}_{3}\right)_{3}\right), 1.55-1.50\left(\mathrm{~m}, 2 \mathrm{H}, T h-\beta-\mathrm{CH}_{2}\right), 1.48-1.40(\mathrm{~m}, 2 \mathrm{H}$, $\left.T h-\gamma^{\prime}-\mathrm{CH}_{2}\right), 1.40-1.30\left(\mathrm{~m}, 4 \mathrm{H}, T h-\mathrm{CH}_{2^{-}}\right), 1.20-1.10\left(\mathrm{~m}, 6 \mathrm{H}, T h-\mathrm{CH}_{2^{-}}\right), 1.20(\mathrm{~d}, J=6.8 \mathrm{~Hz}, 12 \mathrm{H}$, $P h$-CH- $\left.\left(\mathrm{CH}_{3}\right)_{2}\right), 0.89$ (t, J=6.9 Hz, 3H, $\left.T h^{\prime}-\mathrm{CH}_{3}\right), 0.77$ (t, J=6.9 Hz, 3H, Th-CH $\left.{ }_{3}\right)$

${ }^{13} \mathrm{C}-\mathrm{NMR}\left(\mathrm{CDCl}_{3}\right): \delta=166.26,164.88,151.36,146.63,146.52,142.89,141.57,138.44$, $138.16,137.05,135.82,134.83,134.80,132.96,131.95,131.81,131.47,131.42,131.31$, $130.95,130.37,130.31,130.28,129.17,128.22,128.08,127.80,127.62,127.35,127.00$ $124.94,124.03,123.66,122.03,121.96,121.32,121.23,81.71,32.62,32.41,31.53,31.39$, $30.60,30.21,30.08,29.95,29.84,29.16,24.96,23.56,23.41,15.04,14.93$

MS (MALDI-TOF): $m / z\left[\mathrm{M}+\mathrm{H}^{+}\right]=1257$

Elemental analysis: calcd (\%) for $\mathrm{C}_{82} \mathrm{H}_{84} \mathrm{~N}_{2} \mathrm{O}_{6} \mathrm{~S}_{2}: \mathrm{C} 78.31, \mathrm{H} \mathrm{6.73,N} \mathrm{2.23;} \mathrm{found} \mathrm{C} \mathrm{78.14,} \mathrm{H}$ $6.48, \mathrm{~N} 1.93$

\section{4,4'-[(4-\{5'-([N-(2,6-Diisopropylphenyl)]-9-perylenyl-3,4-dicarboxyimide)-3,4'-dihexyl-} 2,2'-bithien-5-yl\}phenyl)imino]dibenzoate (PMI-T2-TPA):

$3(126 \mathrm{mg}, 0.1 \mathrm{mmol})$ was dissolved in a mixture of dry dichloromethane and trifluoroacetic acid. The resulting deep violet solution was stirred at room temperature for 6 hours. After the 
reaction was completed the solvent was removed and the product was dried in vacuum to give

PMI-T2-TPA (115 mg, quantitative) as a black solid. M.p.: 230-231 ${ }^{\circ} \mathrm{C}$

${ }^{1} \mathrm{H}-\mathrm{NMR} \quad\left(\mathrm{CDCl}_{3}\right): \quad \delta=8.67(\mathrm{~d}, J=7.8 \mathrm{~Hz}, \quad 2 \mathrm{H}, \quad$ Pery-1H,6H), 8.53-8.45 $(\mathrm{m}, \quad 4 \mathrm{H}$, Pery-2H,5H,7H,12H), 8.05-7.97 (m, 5H, Pery-8H, HOOC-Ph-2H,6H), 7.70 (d, J=7.8 Hz, 1H, Pery-10H), 7.66 (d, J=7.8 Hz, 1H, Pery-11H), 7.61 (approx. d, J=8.3 Hz, 2H, TPA-Ph-3H,5H), 7.49 (t, $J=7.8 \mathrm{~Hz}, 1 \mathrm{H}, P h-4 H), 7.35$ (d, J=7.8 Hz, 2H, Ph-3H,5H), 7.22-7.15 (m, 8H, Th'-4H, Th-3H, HOOC-Ph-3H,5H, TPA-Ph- $\mathrm{H}, 6 H), 2.86 \mathrm{ppm}\left(\mathrm{t}, J=7.4 \mathrm{~Hz}, 2 \mathrm{H}, T h-\alpha^{\prime}-\mathrm{CH}_{2}\right), 2.79$ (hep, $\left.J=6.8 \mathrm{~Hz}, 2 \mathrm{H}, P h-\mathrm{CH}-\left(\mathrm{CH}_{3}\right)_{2}\right), 2.45\left(\mathrm{t}, J=7.2 \mathrm{~Hz}, 2 \mathrm{H}, T h-\alpha-\mathrm{CH}_{2}\right.$ ), 1.72 (quin, $J=7.6 \mathrm{~Hz}$, $2 \mathrm{H}, T h-\beta$ ' $-\mathrm{CH}_{2}$ ), 1.57 (quin, $\left.=6.8 \mathrm{~Hz}, 2 \mathrm{H} T h-\beta-\mathrm{CH}_{2}\right), 1.50-1.40\left(\mathrm{~m}, 2 \mathrm{H}, T h-\gamma^{\prime}-\mathrm{CH}_{2}\right.$ ), 1.38-1.30 (m, 4H, Th- $\left.\mathrm{CH}_{2^{-}}\right), 1.20-1.10\left(\mathrm{~m}, 6 \mathrm{H}, T h-\mathrm{CH}_{2^{-}}\right), 1.20(\mathrm{~d}, J=6.8 \mathrm{~Hz}, 12 \mathrm{H} \mathrm{Ph-CH}$ $\left.-\left(\mathrm{CH}_{3}\right)_{2}\right), 0.89\left(\mathrm{t}, J=6.8 \mathrm{~Hz}, 3 \mathrm{H} \mathrm{Th}{ }^{\prime}-\mathrm{CH}_{3}\right), 0.77\left(\mathrm{t}, J=6.8 \mathrm{~Hz}, 3 \mathrm{H} \mathrm{Th}-\mathrm{CH}_{3}\right)$

${ }^{13} \mathrm{C}-\mathrm{NMR}\left(\mathrm{CDCl}_{3}\right): \delta=172.35,164.93,152.28,146.63,145.96,142.92,141.67,141.61$, $138.46,138.18,136.97,135.80,134.91,134.77,133.02,132.74,132.26,131.92,131.41$, $131.30,131.23,130.41,130.29,129.15,128.25,128.14,127.78,127.68,127.28,124.97$, $124.56,124.06,123.58,121.97,121.90,121.34,121.25,32.63,32.42,31.54,31.41,30.68$, $30.22,30.09,29.96,29.86,24.96,23.57,23,42,15.05,14.94$

MS (MALDI-TOF): $m / z\left[\mathrm{M}+\mathrm{H}^{+}\right]=1145$

Elemental analysis: calcd (\%) for $\mathrm{C}_{74} \mathrm{H}_{68} \mathrm{~N}_{2} \mathrm{O}_{6} \mathrm{~S}_{2}$ : C 75.57, H 7.73, N 0.77; found C 75.65, H $7.79, \mathrm{~N} 0.85$ 